\title{
La Sociología como profesión: Estrategias de potenciación de la profesión sociológica en el Principado de Asturias
}

\section{Sociology as a Profession: Strategies for Upgrading the Sociological Profession in the Principality of Asturias}

\section{*Ángel Alonso Domínguez}

Departamento de Sociología. Universidad de Oviedo. España/Spain

alonsodangel@uniovi.es

\section{Nerea Eguren Adrián}

Consejería de Servicios y Derechos Sociales del Principado de Asturias. España/Spain

nerea.egurenadrian@asturias.org

\section{Antón González Fernández}

Observatorio sobre Drogas y Adicción a las Bebidas Alcohólicas del Principado de Asturias. España/Spain joseantonio.gonzalezfernandez@asturias.org

Recibido / Received: 12/05/2016

Aceptado / Accepted: 20/08/2016

\section{RESUMEN}

El objetivo del artículo es establecer un debate sobre la situación actual de la sociología como profesión, identificando elementos que permitan clarificar y concretar el rol profesional del sociólogo, que no acaba de encontrar el reconocimiento y el espacio necesario que sí existe para otras disciplinas dentro de las Ciencias Sociales.

El artículo examina, en primer lugar, el contexto global y local en el que se encuentra la profesión. En segundo lugar, analiza los elementos o factores relacionados con la situación de la profesión en tres niveles propuestos (macro, meso y micro). Los retos de la Sociología discurren entre la orientación hacia los problemas de interés público, la búsqueda de la fortaleza grupal, o de un enfoque académico que revalorice la sociología aplicada y descriptiva.

En las conclusiones se aportan ideas operativas y estrategias de actuación respecto al quehacer sociológico para potenciar la profesión.

Palabras clave: Sociología, profesional, Ciencias Sociales, ideas operativas, Asturias.

\section{ABSTRACT}

The purpose of this article is to establish a discussion of the current status of sociology as a profession. We aim to identify those elements which clarify and define the professional role of the sociologist, who is not given the necessary recognition and space awarded to other disciplines within Social Sciences.

The paper, first, examines the global and local context in which the profession exists. Secondly, it analyzes the elements or factors related to the situation of the profession in three proposed levels (macro, meso and micro). The challenges of Sociology run from the orientation through problems of public interest to strengthening the discipline and also establishing an academic approach that revalues applied and descriptive sociology.

The conclusions provide operative ideas and action strategies regarding the sociological endeavour in order to enhance the profession.

Keywords: Sociology, professional, Social Sciences, operational ideas, Asturias.

\footnotetext{
*Autor para correspondencia / Corresponding author: Ángel Alonso. Departamento de Sociología. Facultad de Economía y Empresa. Edificio Departamental, 2. ${ }^{a}$ planta. Campus Universitario del Cristo s/n. 33006 Oviedo.

Sugerencia de cita / Suggested citation: Alonso- Domínguez, A., Eguren, N., González- Fernández, J. A. (2016). La Sociología como profesión: Estrategias de potenciación de la profesión sociológica en el Principado de Asturias. Revista Española de Sociología, 25 (3 Supl.), 171-188. (http://dx.doi.org/10.22325/fes/res.2016.13)
} 


\section{INTRODUCCIÓN'}

En el momento del nacimiento del Colegio de Sociólogos y Politólogos de Asturias (CAPS) (2012) un grupo de profesionales se planteó la oportunidad de abrir un debate reflexivo sobre la profesión sociológica en la situación histórica actual en España y en Asturias. Y esto no solo por el mismo hecho de la creación del Colegio profesional en nuestra Comunidad Autónoma, sino por la opinión mayoritariamente compartida dentro del colectivo profesional de que la profesión se encuentra en un momento que podríamos calificar de incertidumbre e indefinición.

No se trata de una opinión liderada por un grupo y trasladada al resto de miembros en contra del mayoritario parecer. Antes al contrario, se trata de una reflexión planteada desde hace ya algunos años (al menos desde los primeros de este siglo xxl) por otros grupos profesionales del resto de España (Colegio Oficial de Doctores y Licenciados en Sociología y CC Políticas de Navarra 2008a) y ante la que, en consecuencia, la sociología asturiana no ha quedado al margen. Sin ir más lejos, en el XI Congreso nacional organizado por la Federación Española de Sociología (FES), se celebró un simposio denominado La profesión sociológica en España y en el X Congreso se debatieron asuntos profesionales en el grupo de trabajo La Sociología como Profesión. En el trabajo de estos grupos (Gómez-Yáñez, 2010; 2012) y en las lecturas propuestas en el grupo homónimo creado en el seno del CAPS junto con la Asociación Asturiana de Sociología (AAS), encontramos una idea recurrente: la práctica profesional sociológica se encuentra en la actualidad en una situación de inseguridad (valga el psicologismo) profesional. De hecho, nos atrevemos a afirmar que la existencia de tantos artículos y grupos de trabajo sobre el tema son una prueba de cargo sobre las dudas que, sobre su entidad y pertinencia académico-profesional, asaltan en la actualidad al colectivo de profesionales sociólogos.

1 Nota respecto al lenguaje de género: conscientes de la importancia y necesidad de utilizar el lenguaje de género en el presente documento, pero con el fin de evitar la reiteración, se señala que el término "sociólogo/s" se refiere tanto a sociólogo/s como a socióloga/s.
Como prueba de esta situación, al tiempo que se realizaba en Asturias la reflexión colectiva que da lugar al presente documento, se celebraba los días 21 y 22 de noviembre, en la Facultad de Ciencias Sociales de la Universidad de Valencia la Primera Conferencia de Responsables de Sociología de las Universidades Españolas con el objeto de llevar a cabo una reflexión colectiva sobre la situación de la sociología en nuestro país, tanto desde un punto de vista académico, disciplinar y profesional.

Este hecho corrobora la corriente existente en la actualidad dentro de nuestro país sobre la necesidad de reflexionar acerca de la situación académica y profesional de la sociología. Hay que destacar que no somos la única disciplina/profesión que se encuentra en esta encrucijada; ciertamente otras disciplinas y profesiones con mayor recorrido temporal se encuentran en una situación similar sin que hayan emprendido un camino como el que la Conferencia citada y este documento pretenden iniciar. Este es un aspecto que queremos destacar como valioso y positivo desde el inicio de esta reflexión, ya que esta mirada hacia el interior y el exterior consideramos constituye una palanca de cambio y un punto de inflexión en nuestra profesión.

Esta situación es el marco en el que se crea el CAPS, y no parece un trabajo vano comenzar dedicando un tiempo y esfuerzo a pensar sobre la situación en la que se encuentra la profesión, cuáles son sus debilidades, amenazas, fortalezas y oportunidades, cuáles pueden ser algunos de los caminos y de las estrategias a seguir por el CAPS de cara a futuras actuaciones.

\section{DISEÑO Y METODOLOGÍA}

Desde un principio, el proceso de reflexión se guió por dos principios rectores: la participación y el carácter operativo. El principio de la participación buscaba reunir y confrontar las experiencias, opiniones y sugerencias de toda la comunidad sociológica asturiana con el doble objetivo de elaborar un texto plural y representativo al tiempo que ir legitimando y consolidando cada uno de los borradores surgidos de este proceso. El principio de la operatividad se estableció con el fin de obtener un 
documento que sirviera para difundir a otros colectivos las propuestas y demandas de la comunidad sociológica. Esta reflexión debía tener un resultado práctico que facilitase un camino de mejora de la profesión.

Con ambos criterios se estableció una metodología de participación y elaboración de trabajo en torno a tres fases, cuyo calendario se inició en junio de 2013 y finalizó en febrero de 2014.

En primer lugar, utilizando el soporte de comunicación que ofrecieron tanto la AAS como el CAPS, se invitó al conjunto de personas asociadas y colegiadas a la creación de un grupo de trabajo que estableciera los elementos principales del debate e identificara aspectos operativos.

En una segunda fase, se propuso que cada una de las personas integrantes del Grupo de Trabajo elaborase un texto de ideas que incluyera aqueIlos conceptos clave que considera centrales en su planteamiento, con un breve desarrollo de los mismos. Los coordinadores del Grupo de Trabajo realizaron un texto resumen de las propuestas principales para, posteriormente, remitirlo a los miembros del Grupo.

En una fase posterior, se ofreció al conjunto de personas asociadas (y a algunos "evaluadores" externos) la posibilidad de conocer el texto elaborado para que pudieran realizar aportaciones al mismo. Una vez recogidas todas las sugerencias recibidas de la fase anterior, se elaboró un texto definitivo que constituye el marco previo que definirá el documento sobre «La Sociología como Profesión» (para más detalles, ver Anexo 1).

\section{IDEAS EXPLICATIVAS: CONTEXTO GLOBAL Y LOCAL EN EL QUE SE ENCUENTRA LA PROFESIÓN}

Como se ha señalado anteriormente, la profesión parece encontrarse en la actualidad en un momento de incertidumbre, aunque no siempre ha sido así. Durante los años 70 y 80, el contexto socio histórico español de aquellos años quizás favoreció el conocimiento y prestigio de la sociología en los ámbitos académico, mundano 0 popular y profesional en nuestro país, a partir de las demandas de la «nueva» sociedad española
(Del Campo, 2001; Díez Nicolás, 2007; Ortí, 2007, Rodríguez-lbáñez, 2011). Figuras como Salvador Giner, Salustiano Del Campo, Emilio Lamo de Espinosa, Francisco Ayala, Juan José Linz, Amando de Miguel, Manuel Castells, Jesús Ibáñez, Inés Alberdi, Javier Elzo, Julio Pérez Díaz, Carlos Moya, José María Maravall, José Luis Escohotado o José María Sánchez Carrión son parte de un largo etcétera que, cada uno en su ámbito de investigación, docencia o trabajo, destacaron y prestigiaron (y continúan haciéndolo) tanto la perspectiva de conocimiento sociológico como a sus profesionales, situándola en lo más alto de la escala del crédito académico, profesional y popular.

Pero, con la «normalización» democrática de los 90 años, ese vigor que la sociología alcanza en los años precedentes parece perder impulso, y con él el liderazgo profesional, académico y popular alcanzado. Sin embargo, al mismo tiempo, los años 90 fueron el período en el que se crearon más plazas de sociólogos en las Administraciones Públicas y en el que más aumentó el número de facultades y departamentos universitarios (Alvira, 2001). La explicación a esta (al menos aparente) contradicción que supone el inicio del declive del prestigio académico, profesional y popular al mismo tiempo que crecimiento de puestos de trabajo específicos en nuestra materia, puede ser interpretada por un lado como la recogida de los frutos de la siembra y el excelente trabajo llevado a cabo en las décadas anteriores y, por otro, en el desarrollo universitario que vive nuestro país desde finales de los años 80 gracias al impulso autonómico dado a los campus universitarios.

En cualquier caso, en la primera década del siglo xxı la profesión sociológica permanece, a juicio mayoritario de sus profesionales, en esa dinámica gris, de tono bajo en la que se situó en los años 90 , quizás sólidamente instalada en el espacio académico, pero alejada del debate público y profesional, del debate de los procesos y de las ideas sociales, en flagrante abandono de su identidad, que por nacimiento y tradición la sitúa en el centro de los grandes debates históricos de cambio intelectual y social (Machado, 2012; Cock, 2013), algo que resulta particularmente llamativo en el momento actual. 
No deja de ser curioso que la actual débil posición de la profesión sociológica, al menos en nuestro país, coincida con uno de los momentos de tensión, cambio, transformación (que cada cual elija sus adjetivos) de mayor trascendencia de las últimas décadas. Y no solo por lo que está ocurriendo en nuestro país, sino porque lo que está pasando aquí está directamente relacionada con lo que está ocurriendo a nivel europeo y mundial; cambios en el statu quo de la geopolítica mundial (con el crecimiento de China y de los países de la región asiática), cambios socioeconómicos internos en otras grandes regiones como Sudamérica (Brasil, Chile, Venezuela, Perú o Colombia), Rusia y la misma Europa occidental, con el declive y desmantelamiento de los modelos de Estado del Bienestar nacidos después de la II Guerra Mundial, proceso animado al calor de la crisis económico-financiera e implementado a través de las soluciones neoliberales de los gobiernos nacionales de corte conservador y las esferas de poder económico/financiero de la Unión Europea (Beck, 2001). Allí donde se mire sociológicamente hablando tanto a nivel macro, meso 0 micro, fuera o dentro, se están produciendo tal cúmulo de cambios y transformaciones que resulta paradójico que al mismo tiempo la interpretación sociológica tenga escasa presencia.

Al acercarnos al análisis de los elementos que explican esta situación encontramos al menos tres dimensiones o niveles contextuales; una macro estructural, en el horizonte de las grandes corrientes ideológicas y sociales a nivel mundial y europeo, que ciertamente escapan a las posibilidades de actuación de los profesionales sociólogos, pero que debemos de analizar en la fase de identificación de los factores que explican la situación que vive la profesión en la actualidad. Una dimensión meso, intermedia, que explicaría la situación de la profesión sociológica en el contexto de la sociedad española actual. Y un nivel micro, perteneciente al contexto de nuestra comunidad autónoma.

Una vez señalados y analizados los elementos 0 factores relacionados con la situación de la profesión en los tres niveles propuestos (macro, meso y micro), estaremos en condición de plantear estrategias de actuación, prácticas concretas, en el nivel de actuación inmediata del CAPS.

\section{a) Nivel Macroestructural}

\section{El liberalismo económico como perspectiva dominante}

Como dimensión macroestructural, identificamos dentro de las grandes corrientes ideológicas que en cada momento favorecen la visibilidad de determinadas líneas de pensamiento, el resurgimiento del discurso económico neoliberal que tuvo lugar en la década de los 80 en USA e Inglaterra, liderados por Reagan y Thatcher, y que llegaría en los 90 a España y otros países europeos como Alemania, Italia, Francia o Portugal, con los gobiernos conservadores, como uno de los factores que explican el paso a segundo plano de la visión sociológica.

Como sabemos, la sociología nació y creció primero estructural y luego crítica. En la actualidad la corriente mundial del pensamiento dominante es económica y psicologista, relegando hacia una posición periférica en el «jueg0» de las disciplinas científicas el discurso sociológico que replica ambos supuestos. La sociología crítica se desarrolló como reacción a la ciencia positivista y esto significa un interés no solo por la comprensión del mundo social, sino también por el cambio, por la transformación social y política (Machado, 2012; Lichtenstein, 2013). Y frente a esta perspectiva clásica de la sociología, el discurso económico neoliberal fundamenta y favorece las explicaciones sobre la realidad social desde la perspectiva economicista y psicologista (individualista), en las que la situación que realmente ocupa cada persona dentro de la estructura social tiene poco que ver con su contexto de socialización (de dónde viene), sino que se explica por su proyecto personal (hacia dónde quiere ir) tanto desde el punto de vista económico como psicológico, por sus cualidades y actitudes (la responsabilidad de lo que te pasa y de cómo te sientes es individual), y que minimizan como irrelevante el factor del contexto, negando 0 reduciendo la importancia (empíricamente comprobada) del entorno social no ya sobre la situación sino sobre el proyecto personal de cada uno. Como sabemos «de dónde vienen las personas» determina en alto grado no solo "hacia dónde quieren y pueden ir», sino por supuesto, «a dónde terminan efectivamente llegando». 
La evidencia empírica sobre el peso que el contexto social tiene como factor que correlaciona fuertemente con las conductas y situaciones 0 posiciones sociales de las personas es profusa en todos los ámbitos, de tal forma que existen centros específicamente dedicados a resumir la evidencia de la investigación social internacional, como, por ejemplo, la Campbell Systematic Reviews sobre los efectos de las intervenciones de la delincuencia y la justicia, la educación, el desarrollo internacional, y el bienestar social; sobre la relación existente entre la clase social de pertenencia y las probabilidades de disfrutar de una larga carrera formativa y de alcanzar un buen puesto de trabajo (Grundiza y López-Vilaplana, 2013); 0 sobre el famoso concepto de «capital social» entendido como «normas y redes que facilitan la acción colectiva» (Woolcock, 2001) en relación a la disponibilidad de mayores y mejores oportunidades para aquellas personas que cuentan con más apoyos u oportunidades sociales (redes sociales y recursos institucionales).

En clave de autocrítica, y como complemento a lo señalado sobre la hegemonía actual del discurso económico liberal para explicar la realidad, Gómez-Yáñez señala en sus comentarios a este texto que "La sociología ha abandonado el mercado. Por alguna razón, la sociología decidió que el mercado era homo económico... En términos prácticos esto es grave, porque ha alejado a los sociólogos de la lógica económica: consideran el marketing como una maldición en vez de como sociología aplicada, no saben de economía, etc.».

\section{b) Dimensión meso}

En el discurso de la autocrítica, como elemento meso, algunos de los colegas que han escrito sobre la cuestión están de acuerdo en que buena parte de la responsabilidad de la pérdida de presencia profesional en nuestro país la tiene el propio colectivo de sociólogos y ello por varias cuestiones.

\section{Falta de conexión con el debate público}

Por un lado, se señala la falta de un discurso sociológico relevante sobre temas sociales de interés para los ciudadanos (Tezanos, 2001; Pérez-Yruela, 2007a; Fernández-Alcalde, 2010); buena parte de la producción sociológica actual se produce y se orienta en y hacia el entorno académico más que a las preocupaciones sociales más notables, 0 al menos esto parece ser lo que llega a la opinión pública.

El conocimiento sociológico desde el inicio de la crisis en nuestro país ha participado del debate público y político pero no así los sociólogos. Movimientos sociales como el 15-M o Stop Desahucios han puesto sobre la mesa grandes temas sociales clásicos y modernos, pero es notable la escasa presencia de profesionales de la sociología que han participado en el debate experto, especialmente significativa en momentos en los que el discurso sociológico debería de destacar sobre otros enfoques académicos, teóricos y profesionales.

El conocimiento experto, «la imaginación sociológica» (Mills, 1969), o lo que en términos más actuales Gómez-Yáñez (2012: 129) denomina la «perspectiva productiva, ver lo que otros no ven» destaca por su ausencia en un contexto social particularmente necesitado de explicaciones sociológicas, y cuando se dan, frecuentemente son emitidas por especialistas de otras disciplinas. El aumento de la pobreza y el debate sobre la creciente desigualdad social en relación con el modelo de estado; el impacto que las tecnologías de la comunicación están teniendo en el nacimiento y desarrollo de los movimientos sociales de contestación, el comportamiento de las élites o la cultura política de nuestro país en relación al modelo de gestión de los recursos públicos y su impacto en el modelo político y social, son temas de enorme calado, de gran actualidad y en los que los profesionales de la sociología no participan cuando su formación les cualifica especialmente para ello.

\section{La popularización del Conocimiento Sociológico}

La popularización del Conocimiento Sociológico ha sido en ocasiones utilizada como explicación del declive de la sociología como profesión; la paradoja sociológica es que su punto de vista ha permeado el discurso profesional del resto de las ciencias sociales, el discurso político oficial, el de los medios de comunicación y el del «conocimiento mundano», de tal forma que la voz de los profesionales de la sociología ha perdido peso como referente a la hora de buscar una explicación sobre «lo que pasa».

El hecho de que el discurso y la terminología sociológica hayan traspasado otros ámbitos de 
conocimiento es comprensible, dada la necesidad de explicaciones e interpretaciones de la compleja realidad social y, en todo caso, es más un éxito que un demérito para la profesión sociológica. Nos atrevemos a afirmar que ese no es el problema, sino la colonización del espacio profesional sociológico por personas no expertas, no adiestradas específicamente en este ámbito de conocimiento, lo que ha trivializado y desdibujado el discurso especializado (Béteille, 2013) apartándolo del conocimiento científico, empíricamente fundado y sustituyéndolo frecuentemente por otros discursos débiles, ideológicamente contaminados, a-científicos, que no pasan de la mera opinión, contribuyendo de esta manera a su desprestigio.

Ligado a la popularización de la perspectiva sociológica, la aparición de especialidades profesionales en competencia con la profesión sociológica ha contribuido también a restar visibilidad a la profesión. Así, a especialidades académico-profesionales tradicionalmente en «competencia» con la sociología como pueden ser la Antropología Social y Cultural, la Etnografía, las Ciencias Políticas, la Economía o la Demografía, en las últimas décadas se han sumado nuevas especialidades que han ocupado o colonizado su espacio profesional. En esta categoría se sitúan titulaciones como Educación Social, Relaciones Laborales, Trabajo Social, Ciencias del Trabajo, Investigación y Técnicas de Mercado, Ciencias y Técnica Estadísticas, etc., que poco a poco han contribuido a restar espacio profesional a nuestro ámbito de conocimiento.

\section{Pérdida de profundidad y falta de innovación}

Los «padres» de la sociología tienen su lugar en el pensamiento actual (Lichtenstein, 2013) pero a la falta de interés por la profesión sociológica ha contribuido también la ausencia de creación teórica innovadora dentro de la propia sociología, que vaya más allá de las continuas revisiones de los clásicos. Se ha mostrado de esta manera ante la comunidad científica, profesional y ciudadana como una producción teórica agotada o falta de ideas nuevas.

Sin embargo, en el ámbito internacional, autores contemporáneos como Beck (2002) o Bauman (1999) han acuñado conceptos y modelos innovadores de interpretación de la realidad social que han supuesto una renovación y actualización de la teoría y la investigación sociológica aplicada. En cualquier caso, estas innovaciones no llegan con la adecuada fluidez al debate público ni son empleadas por la comunidad de sociólogos más allá del entorno académico, donde sí es apreciada y valorada.

Es curioso, también, que la profesión sociológica dude sobre su pertinencia o relevancia como conocimiento experto dentro de las ciencias sociales, cuando los premios Príncipe de Asturias en su corta vida han otorgado el galardón a un total de 8 profesionales de la sociología. Han sido merecedores de este premio en la categoría de Ciencias Sociales la recién galardonada Saskia Sassen, Ralf Dahrendorf, Jürgen Habermas, Anthony Giddens, Luis Díez del Corral y Juan José Linz. Además de Zygmunt Bauman y Alain Touraine, que lo fueron en Comunicación y Humanidades. Es este un hecho que debería ayudarnos a reflexionar sobre si la vitalidad de la profesión corre en paralelo con el reconocimiento académico de la disciplina.

\section{Falta de rigor teórico y metodológico}

También se denuncia en muchos casos la falta de rigor, de profundidad teórica, metodológica de las obras producidas o editadas (Subirats, 2007), y la escasa 0 nula presencia internacional de la sociología española puede ser una buena muestra de ello (Pérez-Yruela, 2011). Es sin duda una responsabilidad que nos atañe directa y particularmente, ya que nos compromete como profesionales «creíbles», si de reclamar atención y respeto se trata.

Hemos de reconocer que en algunas ocasiones, atraídos u obligados bien por la urgencia, bien por la necesidad, la precariedad e inestabilidad laboral 0 , por qué no decirlo, por la debilidad consustancial de la condición humana ante el halago personal o la anhelada fama o popularidad, algunos de nosotros hemos podido sentirnos tentados a involucrarnos en trabajos, investigaciones, redactar artículos 0 a participar en charlas como expertos sin responder al perfil necesario.

Dentro de este factor, pero ligado también a otros que explican la debilidad de la profesión en la actualidad (como los ya citados abandono del «mercado», el marketing 0 el mercado de trabajo en manos de los economistas, la pérdida de profundidad y falta de 
innovación e incluso el analizado a continuación escaso prestigio de la sociología aplicada y descriptiva) aparece la deficiente formación que la profesión tiene (en general) en un ámbito fundamental para el conocimiento social como es la matemática estadística, aspecto considerado durante décadas como de segundo orden en un currículum académico universitario marcado en exceso por la «teorización», punto débil de la profesión sociológica que necesariamente hay que corregir dentro de una estrategia de revalorización, tal y como recoge la Conferencia de Responsables Académicos del Área de Sociología de las Universidades Españolas².

\section{Escaso prestigio de la sociología aplicada y descriptiva}

Las administraciones públicas, las empresas, las organizaciones políticas, organismos de todo tipo, utilizan constantemente investigaciones basadas en datos empíricos para ayudar en la toma de decisiones, con el objeto de ir más allá de la opinión y la ideología respecto a sus actuaciones. En este tipo de estudios la explotación de bases de datos secundarias, registros administrativos y las encuestas son herramientas de investigación aplicada y descriptiva útiles para reducir el margen de error en la toma de decisiones y en la mejora de la relación coste/efectividad.

Sin embargo, esta rama de la sociología es vista en ocasiones por una parte del colectivo profesional como una práctica de «segundo nivel» basando quizás su opinión en el empleo de técnicas de investigación y modelos teóricos alejados de la sofisticación 0 excesiva elaboración propias de la sociología más teórica (Valdera, 2011).

Parece indiscutible la falta de interés, 0 incluso de aprecio, que dentro de la sociología académica despierta la producción sociológica aplicada y descriptiva, desarrollada en el mundo empresarial y de la administración (Gómez-Yáñez, 2012). Sin embargo esta sociología aplicada hace posible la

2 Celebrada en la Facultat de Ciències Socials, Universitat de València el 21 y 22 de noviembre de 2013. Documento disponible en: http://www.fesweb.org/uploads/files/conferencia-responsablesacademicos/acta-reunion-noviembre-2013.pdf toma de decisiones, combinando la flexibilidad en la utilización de métodos, técnicas e instrumentos; en definitiva una "adaptación constructiva a diferentes alternativas de trabajo y desafíos" (Machado, 2012: 119) que la convierten en una de las líneas de trabajo profesional de mayor relevancia actual y futura (Pérez-Yruela, 2007a).

Prestigiar y valorar desde dentro de la profesión esta rama de la sociología aplicada, revalorizarla mediante las acciones adecuadas, sería hacerlo del conjunto de la profesión, y también de cara a los ciudadanos, las empresas y los entes públicos, reforzando el vínculo y la cercanía con todos ellos (Tezanos, 2001; Pérez-Yruela, 2007b; Gómez-Yáñez 2012).

\section{Marco de juego legal de los colegios profesionales}

En este contexto aparece como factor añadido una nueva amenaza; la modificación de la ley de colegios profesionales que, en la dinámica de liberalización profesional del espacio económico de la Unión Europea, aparece como un riesgo más de dispersión del perfil profesional del sociólogo. El concepto de «profesional sociólogo» puede ir a menos a favor de la «práctica sociológica». Evidentemente, esto no afecta al conocimiento sociológico, ni al concepto mismo de sociología, pero sí al de sociólogo definido frente a otros profesionales asociados al campo teórico del conocimiento social.

Por otra parte, la atomización de los sociólogos (Alvira, 2001; Machado, 2012) estaría produciendo en determinados ámbitos una falsa sensación de distanciamiento del trabajo sociológico. Nuestra profesión es rica, compleja y diversa pero la «soledad» del sociólogo potencia esta visión de renuncia, de trabajar al margen de la profesión que se refleja en las pocas encuestas que existen en España sobre el perfil profesional del sociólogo (Colegio Oficial de Doctores y Licenciados en Sociología y CC Políticas de Navarra 2008a; 2008b) e impide la introducción de regulaciones normativas que delimiten el ámbito del ejercicio de la profesión (Machado, 2012).

Tampoco desde las propias instituciones profesionales (universidades, colegios, asociaciones) se presta la suficiente atención a las dinámicas de sus graduados, sus competencias, su vocación 0 interés 
instrumental, ni a su satisfacción con la elección realizada, más allá del conocimiento por medios informales; una falta de interés, en definitiva, que dificulta la mejora de la profesión (Cipriani, 2012). Las instituciones profesionales de la Sociología deben potenciar la visibilización de la profesión, constituyéndose en instrumentos de presencia y alianza con otras ciencias sociales (Guilló, 2007). En este sentido, la constitución de pleno derecho del CAPS debería significar una buena oportunidad para la integración de los sociólogos asturianos, así como para la defensa del estatus profesional, de fortalecimiento de un «espíritu de cuerpo» que se desvanece más allá de la Universidad (Fernández-Alcalde, 2010).

\section{c) Nivel micro}

Los escasos profesionales sociólogos que actualmente trabajamos en nuestra Comunidad Autónoma nos encontramos con un entorno poco favorable y con limitaciones importantes, y no solo por las intrínsecas a una región de reducido tamaño, sino porque el peso que tienen en entornos profesionales como la Universidad o la Administración regional (que podrían servir de espacios de visualización al resto de la sociedad), es muy pequeño. En el primer caso por no existir facultad propia y en el segundo porque no existe perfil profesional definido como tal a nivel de grupo, cuerpo 0 escala, lo que a nuestro juicio influye en la presencia de la profesión en ámbitos en los que tendría perfecto encaje. Estas y otras debilidades se detallan a continuación.

\section{Comunidad Autónoma de tamaño pequeño}

En el juego de los grupos sociales evidentemente el tamaño importa; el volumen de los grupos se relaciona con su peso en términos de poder y capacidad de influencia. Una comunidad autónoma pequeña como Asturias no facilita la existencia de un grupo profesional como el nuestro que, por sus propias características, ofrece productos y servicios a un número de clientes reducido; organizaciones 0 empresas de media o pequeña dimensión, apenas un millón de habitantes, administración regionales y locales de tamaño acorde, lo mismo que su Universidad (cuestión que trataremos a continuación) y un entramado empresarial de herencia industrial que ni por orientación ni por tamaño, es capaz de absorber ni a un gran número de profesionales sociólogos ni a los productos que estos ofrecen.

Con este «techo» cuantitativo, la pregunta relevante que nos planteamos es si la presencia de la profesión en Asturias está por debajo de la capacidad de carga del entorno. Para responder a dicha cuestión podemos tomar como ejemplo el número de sociólogos en la administración, de cuya observación es fácil concluir que la capacidad de carga de profesionales sociólogos del mercado de trabajo de nuestra región tiene aún recorrido al alza. Si hacemos un sencillo ejercicio aritmético para corroborar cuantitativamente esta afirmación, de los cerca de 60000 trabajadores existentes en Asturias en las diferentes administraciones públicas en $2012^{3}$ (del Estado, administración regional, local y universidad de Oviedo) cerca de 35700 pertenecían a la regional de los que, solamente $7(6+1$, ya que una se encuentra en un organismo autónomo) son de plazas de sociólogo, lo que porcentualmente significa el $0,016 \%$ de todos los trabajadores de la administración regional. Aumentar este microscópico porcentaje a un 0,10\% significaría multiplicar por 6 las plazas de sociólogos actuales hasta los 36 y alcanzar el 0,25\% implicaría llegar a cerca de 90 plazas de sociólogos en toda la administración regional.

\section{Presencia académica limitada}

A la vista del vigor que ha tomado la profesión en aquellas comunidades autónomas en las que existen estudios de sociología, la falta de una facultad, de unos estudios específicos en nuestra materia ha restado visibilidad a la profesión, dándoselo a aquellas que sí disponen de ellos.

Por otro lado, si como hemos señalado, en el contexto socio-histórico actual la perspectiva económica domina el espacio profesional de las ciencias sociales y particularmente el de la sociología, la situación en Asturias, con el Departamento de Sociología formando parte de la estructura organizativa de la Facultad de Economía y Empresa limita las posibilidades de desarrollo de la profesión fuera de la Universidad.

3 Ministerio de Hacienda y Administraciones Públicas. http://www.minhap.gob.es/es-ES/Paginas/Home. aspx 
En cualquier caso, no entra dentro de la estrategia de futuro de la Universidad de Oviedo crear una facultad de sociología, ni unos estudios específicos en la materia, y en el contexto actual de su oferta de estudios hemos de valorar como un factor de fuerza para la profesión en Asturias la existencia de los estudios de sociología con la categoría de departamento dentro de una facultad del tamaño cuantitativo y del prestigio académico de la Facultad de Economía y Empresa, lo que debe de ser aprovechado como una oportunidad para el desarrollo de la profesión, para reorientar la sociología hacia la economía, el marketing y las oportunidades en gestión de las empresas: publicidad, comunicación, etc. (Gómez-Yáñez, comentarios al texto).

\section{Presencia en las administraciones públicas: escasa y con indefinición de funciones}

Otro ámbito en importancia en cuanto a presencia profesional sociológica lo constituye la administración pública, en concreto la administración autonómica en la que se observan notables problemas. En primer lugar, es cuando menos sorprendente que en ámbitos administrativos regionales como la educación, el urbanismo o los servicios sociales en las que el perfil profesional del sociólogo es particularmente adecuado, no exista en la actualidad ninguna plaza de sociólogo. En segundo, la indefinición de la profesión reflejada en la ausencia de cuerpo o escala de sociólogos, da como resultado una ausencia de funciones y contenidos a desarrollar al tratarse de una profesión sin ordenar ni definir. La diversidad 0 amplitud de enfoque propia de la disciplina tampoco ayuda a esta definición de funciones ya que, en ocasiones, es entendida como dispersión o falta de concreción y convertirse en debilidad; sin embargo, entendida como versatilidad, constituye una fortaleza (Federación Española de Sociología, 2013). Sin duda, que la profesión sociológica carezca de definición dentro de la administración regional es un factor relacionado con el escaso número de plazas de sociólogo existentes en la actualidad (que ya hemos relacionado). En realidad probablemente sean factores mutuamente influyentes; el escaso número anima a que no sea definida como profesión dentro de la relación de puestos profesionales de la ad- ministración regional y esto influye a su vez en que no sea una profesión demandada en los diferentes servicios o secciones de la administración regional, limitando su número y sus posibilidades de crecimiento cuantitativo.

\section{Intrusismo profesional: falta de profesionales de la sociología en organismos propios de su ámbito de conocimiento}

Ya hemos señalado la llamativa ausencia de plazas de sociólogos en ámbitos de la administración regional particularmente adecuados para el perfil profesional del sociólogo y hemos citado casos tan sorprendentes como los servicios sociales (¿cómo se puede explicar que el brazo de la administración regional que trabaja sobre la pobreza y la exclusión social no disponga de una sola plaza de sociólogo?) y la educación, pero también podríamos hablar en los mismos términos del ámbito de la juventud y de casos igual de flagrantes como el del extinto Instituto Asturiano de Administración Pública, los responsables del Plan de Estadística, etc., ámbitos propios del quehacer sociológico en los que no se encuentra ningún profesional trabajando.

Particularmente llamativo es el caso de la Sociedad Asturiana de Estudios Económicos e Industriales, SADEl, que como todos sabemos y más allá de lo que su propio nombre indica y su propósito fundacional, con el paso del tiempo ha extendido sus actividades a la recogida y análisis y elaboración de información de tipo no solo económico, sino demográfico, social, censal, y que sin embargo no cuenta en su plantilla con ningún profesional de la sociología.

Evidentemente, si no existen plazas de sociólogo en espacios de actividad profesional de la administración regional a los que nuestro perfil se ajusta absolutamente, está claro que la visibilidad de la profesión está muy limitada para otros espacios de actividad en términos de hacer patente el valor que es capaz de aportar la visión sociológica a los servicios y productos. Por ese motivo, lograr que la administración regional cree un perfil profesional de sociólogo y que incorpore profesionales en aqueIlas áreas y organismos que constituyen su campo «natural» de conocimiento, debe de constituir una prioridad dentro de las estrategias de trabajo del colectivo profesional. 


\section{Escasa presencial profesional en el debate público/social}

En los últimos años se ha hecho un importante esfuerzo por parte de la AAS, como se verá en las fortalezas, por implicar a los profesionales en el debate público y en este sentido hemos de reconocer los logros conseguidos. Sin embargo la presencia de la voz sociológica en los debates de actualidad puede ser aún más significativa, y por tanto, la implicación de los miembros de la AAS en esa labor debe de ser aún mayor, evidentemente cada uno/a en su ámbito de conocimiento 0 estudio.

Todas dificultades mostradas tienen, sin embargo, su contrapunto en sólidos elementos que permiten el desarrollo de un trabajo de consolidación y visibilización de nuestra profesión. La trayectoria de la propia AAS y el futuro del CAPS son dos vehículos de trabajo colectivo a través de los que operativizar la propuestas sugeridas en este documento.

Como ya venimos señalando, el mismo hecho de plantear el debate sobre la situación de la sociología como profesión constituye una herramienta de cambio sobre la situación planteada. Otras fortalezas a destacar serían las siguientes:

\section{El Departamento de Sociología de la Universidad de Oviedo se encuentra en la élite de la disciplina}

El mayor número de profesionales de la sociología asturiana (más de una veintena) se encuentra trabajando en la Universidad, en el recientemente creado (2008) Departamento de Sociología. A pesar de su juventud, dicho departamento se encuentra entre los grupos científicos más cualificados de la Universidad de Oviedo y en la élite nacional de su disciplina, en la que ocupa el quinto puesto según la 4. ${ }^{\text {a }}$ edición (mayo de 2013) del «Ranking I-UGR de Universidades Españolas» ${ }^{4}$, y mejorando su posición respecto a ediciones anteriores.

$4 \quad$ El I-UGR es el ranking nacional de las universidades españolas públicas y privadas con mayor prestigio, ya que está basado en la investigación publicada en las revistas internacionales de mayor impacto y visibilidad.
Por tanto, y aunque históricamente haya contado con una presencia académica limitada, tal y como se apuntaba en las debilidades, los sociólogos asturianos están consiguiendo que su obra e investigación trascienda en el ámbito universitario. Aun así, sería necesaria una mayor colaboración y diálogo entre el ámbito académico y el ámbito profesional (Federación Española de Sociología, 2013). Como hemos señalado en el apartado anterior, y asumiendo que la Universidad de Oviedo no va a potenciar el desarrollo de la sociología como especialidad académica, deberíamos de valorar otras posibilidades de crecimiento de la profesión a partir del departamento y de los profesionales que en el trabajan.

\section{Tradición asociativa}

El proceso de reflexión aquí reflejado ha sido posible de realizar al existir un foro profesional, la AAS, a través del que vehiculizar las inquietudes de un grupo de profesionales asociados a la misma. La AAS cuenta con más de 30 años de actividad continuada y ha servido como lugar de encuentro y debate del tema que nos ocupa. La organización asociativa ha facilitado el desarrollo de una metodología participativa y secuencial, que sin ese soporte hubiera sido difícil llevar a cabo. Bajo el patrocinio de la AAS se han organizado congresos, encuentros, mesas redondas, conferencias o seminarios, también de forma conjunta con otras entidades, en su afán de crear redes de trabajo dentro de nuestra comunidad.

También, en los últimos años, la AAS ha buscado tener una mayor presencia e influencia en la sociedad asturiana, y en ese sentido se cerró un acuerdo de colaboración con el diario El Comercio mediante el cual la sociología ha ganado presencia y visibilidad mediante la publicación de artículos de opinión y blogs firmados por sociólogos asturianos.

\section{Creación del Colegio Asturiano de Ciencias Políticas y Sociología}

Esta tradición asociativa, de carácter más académico, se ha visto reforzada a través de la creación del CAPS como entidad defensora de los intereses profesionales de la comunidad asturiana. Por primera vez nuestra comunidad autónoma cuenta 
con un Colegio profesional que integra a politólogos y sociólogos. La Conferencia de Responsables Académicos señala en sus conclusiones la importante función del Colegio profesional, actualmente en proceso de ajuste al nuevo marco normativo previsto (Federación Española de Sociología, 2013).

\section{Sociedad abierta y movilizada}

Frente a otras regiones, Asturias cuenta con una importante tradición de pensamiento social ${ }^{5}$ y acción ciudadana. Este contexto, unido al momento social y económico que vivimos, debe ser un contexto que facilite la difusión del pensamiento sociológico ayudando a interpretar la realidad que vivimos desde un ángulo de reflexión científica y profesional.

\section{Premios Príncipe de Asturias}

Como se ha señalado, los premios Príncipe de Asturias en sus modalidades de Ciencias Sociales y Comunicación y Humanidades han premiado a una relación de notables sociólogos en varias ediciones. Este hecho visibiliza y pone en valor el pensamiento sociológico dentro de las sociedades actuales. La importancia mediática de estos premios permite una visibilización de la Sociología única en nuestro país.

\section{DISCUSIÓN: IDEAS OPERATIVAS PARA POTENCIAR LA PROFESIÓN EN ASTURIAS. ¿QUÉ HACER?, ¿HACIA DÓNDE IR?}

La explicación meso, que nosotros compartimos, resulta plausible para el análisis de los factores explicativos de la actual situación de la profesión. Sin embargo es necesario pasar del análisis a las propuestas de actuación, con el fin de revertir 0 al menos intentar combatir los factores que afectan a la profesión negativamente y al mismo

$5 \quad$ Históricamente Asturias ha albergado a pensadores de gran impacto intelectual; desde Feijoo y Jovellanos (s. xvill) pasando por el conocido como «Grupo de 0viedo» (profesores universitarios que a finales del xIx pretendieron dotar a la Universidad de Oviedo de un programa pedagógico e investigador que permitiera renovar el ideal universitario) hasta la obra de Gustavo Bueno en la actualidad. tiempo, potenciar los positivos, que obvia decirlo, también hay.

En esta situación de la profesión sociológica, y en el marco de las reflexiones hechas por nuestros colegas destaca la hecha por Pérez-Yruela (2007b: 14) que defiende que pese a «la paradoja o el declive citados, es posible un retorno de la sociología, en el sentido de un nuevo periodo de recuperación de su visibilidad y reconocimiento público» (y académico y profesional añadimos nosotros) y nos advierte de que «los sociólogos no debemos desaprovechar esa oportunidad». ¿Es un visionario que ve lo que los demás no alcanzan a vislumbrar?

El momento actual, en un mundo sujeto a cambios estructurales, crisis, revoluciones e inseguridades, resulta muy propicio para el quehacer sociológico. Existen indudables paralelismos con el contexto social en que surgió la Sociología en el siglo xix y, por tanto, enfrentamos una época atractiva para el resurgir de la profesión (Alvira, 2001). Resulta interesante en este entorno de incertidumbre en el que parecen navegar el resto de reflexiones, encontrar voces que se atrevan a señalar claramente direcciones o vías por las que la sociología del s. XXI puede caminar para salir de la periferia y volver a ocupar posiciones de mayor visibilidad y prestigio social. Pero se requiere otra forma más activa de hacer sociología; denunciar hechos, proponer medidas, involucrarse en los cambios sociales. En definitiva, como dice Guilló (2007: 87), pasar de «la Sociología precaria y clandestina a la Sociología crítica y transformadora».

A juicio de Pérez-Yruela (2007 b; 2011) los factores que en el siglo Xxı posibilitan el renacimiento de la disciplina son los siguientes:

I. La creciente demanda social de microdatos. La complejidad del mundo actual y en particular la sobre-información existente, hace más necesaria que nunca la competencia sociológica para analizar, sintetizar y simplificar el laberinto de la realidad social en conceptos e ideas explicativas fácilmente comprensibles. El conocimiento social experto es necesario porque reduce la confusión y el ruido que llega de todas partes y a todas horas, porque hace 
comprensible la realidad existente, dotándola de sentido y orden. En esta función, el profesional de la sociología ha sido entrenado y dotado de las herramientas teóricas e instrumentos metodológicos para instrumentalizar el conocimiento sobre la realidad y ponerlo al servicio de la sociedad misma.

II. La necesidad de innovación social para resolver 0 afrontar los retos del s. XxI. El momento socio-histórico actual se caracteriza por los cambios orgánicos que están afectando no solo las estructuras sociales, económicas y políticas sino al orden internacional conocido hasta la fecha. Es un cambio que afecta a todo y a todos (desde los fundamentos de legitimación de la democracia, las estructuras socioeconómicas hasta el medio ambiente global 0 la organización de la escala social) y en este contexto la sociedad, los grupos, las personas, necesitamos profesionales expertos que dediquen tiempo y esfuerzos a pensar y a proponer soluciones teóricas y prácticas para afrontar esos nuevos/viejos retos «glocales» (Robertson, 1992).

III. La necesidad que tiene la nueva función pública de conocimiento social profesional (sociología aplicada). En los años 80 los modelos teóricos y los presupuestos prácticos en que se fundamenta la función pública tradicional fueron renovados ante la necesidad de responder y hacer frente a los cambios políticos, sociales y tecnológicos habidos en las democracias occidentales en las décadas precedentes.

IV. Desde mediados los 90 el concepto de «formulación de políticas basada en datos contrastados» definidas como aquéllas que son fruto de un enfoque que «facilita la adopción de decisiones fundadas respecto a políticas, programas y proyectos» (Davies, 2004) se ha erigido como el nuevo estándar de referencia en el terreno de la gestión pública. En este enfoque, la perspectiva sociológica demuestra todo su potencial e idoneidad, ajustándose como perfil profesional mejor que otros a las necesidades de esta nueva función pública. Prueba de esta idoneidad en la nueva administración pública ha sido la proliferación de los observatorios o la redacción de libros «blancos» para analizar un problema desde distintas perspectivas y mejorar el proceso de toma de decisiones (GómezYáñez, comentarios al texto).

V. Popularización de la perspectiva sociológica. Como hemos señalado (en esto hay consenso también), y por eso precisamente, se advierte de la obligación de ser rigurosos y orientarse hacia temas relevantes para la sociedad.

A partir de aquí se apuntan dos retos concretos:

I. Necesidad de revalorizar la sociología aplicada y descriptiva (Pérez-Yruela, 2007b), usada en la toma de decisiones basadas en datos empíricos, más allá de la opinión 0 la ideología.

II. Corporativización activa de la sociología, cuestión defendida abrumadoramente por toda la literatura sobre la profesión sociológica (Tezanos, 2001; Burawoy, 2005; Subirats, 2007; Fernández-Alcalde, 2010; Pérez-Yruela, 2011).

Protección, unión y observación significan corporativización, si se quiere, pero no «cierre profesional» ni, desde luego, aislamiento de otras ciencias, pues el oficio de sociológico implica diversidad y complejidad. La Sociología debe relacionarse e interactuar con otras ciencias, especialmente, claro está, con las sociales (Alvira, 2001; Machado, 2012) si no, afrontaremos un serio riesgo de aislamiento. Se puede reivindicar la mirada especializada de la Sociología pero teniendo en cuenta que los problemas sociales/colectivos necesitan, cada vez más, un enfoque multidisciplinar. Una sociología «abierta también a enriquecerse con las aportaciones que otros puedan hacer» (Pérez-Yruela, 2007b: 14) que se base en argumentos plausibles y operativos.

\section{ESTRATEGIAS DE ACTUACIÓN PARA POTENCIAR LA PROFESIÓN EN ASTURIAS}

De acuerdo al análisis y a las propuestas revisadas se plantea operativizar las conclusiones extraídas bajo la forma de propuestas de actuación lo más concretas posibles que puedan servir como líneas de acción 0 estrategias a seguir en el trabajo del CAPS en el ámbito de nuestra comunidad autónoma. 
a. Opinemos, pronostiquemos, salgamos a la palestra, tengamos iniciativas, generemos debates. Proponemos continuar y fortalecer la labor ya iniciada desde la AAS de participación en medios de comunicación sobre temas que interesen a los ciudadanos. La línea de colaboración abierta con el diario El Comercio es un ejemplo de esta estrategia de acercamiento y visualización de la profesión a través de los medios de comunicación. Hacer este ofrecimiento como CAPS, a otros medios de prensa, radio y TV de Asturias mediante una oferta de disponibilidad de participación, concretándola mediante la disposición de profesionales especializados en diferentes ámbitos de potencial interés para los medios y la sociedad en general (demografía, desarrollo territorial, empresas, educación, desigualdad social, servicios sociales, sanidad, drogas, medio ambiente, participación política, etc.).

b. Orientémonos a los problemas de interés público. Relacionado con el apartado anterior, el CAPS debería elaborar un Programa Anual (o semestral) de actividades de relación con la sociedad en forma de charlas-coloquio en foros públicos en los que tratar temas de actualidad desde la óptica sociológica, si fuera posible, incorporando a estas actividades a sociólogos especializados, que hayan investigado y escrito sobre el tema en cuestión.

c. Agrupémonos en equipos de investigación e innovación social aplicada. Esta propuesta, podría materializarse con la creación de un Equipo de Investigación e Innovación Social Aplicada que, impulsada desde el CAPS y vinculando a instituciones como la Universidad de Oviedo o la administración pública, explore vías de investigación y haga propuestas de actuación concretas de intervención social en torno a problemas sociales relevantes.

d. Participemos activamente en la corporativización sociológica. La participación activa en la defensa de los intereses de la profesión sociológica puede ser entendida en negativo como un cierre pretencioso sobre nosotros mismos con la intención de delimitar un espacio exclusivo de conocimiento y competencias ante el intrusismo profesional, pero también en positivo como el aumento de la solidaridad interna con los colegas de profesión y como el cuidado, respeto, aprecio y rigor en el desempeño de la profesión misma. El CAPS debería elaborar una estrategia en este sentido, detectando los espacios 0 ámbitos profesionales en los que se considere que los sociólogos ofrecen diferencias competitivas frente a otros profesionales, elaborando Fichas Técnicas por ámbito de actuación 0 entorno profesional (público y privado) y estableciendo un calendario de visitas para, a modo de labor comercial, «vender» la figura del sociólogo en esos ámbitos profesionales (administraciones públicas, organismos públicos y privados, organizaciones empresariales 0 sindicales).

e. Abundemos/incidamos en la necesidad que tienen tanto las empresas como la nueva función pública de conocimiento social profesional. Relacionado con la propuesta anterior, uno de los ámbitos de especial interés es sin duda alguna elaborar un plan de trabajo específico con la Administración, tanto a nivel regional como local. Un objetivo concreto a conseguir sería que la Administración del Principado de Asturias oficializara la existencia de un perfil profesional de sociólogos (cuerpo o escala) de la misma forma que ya existe el de psicólogo 0 el de economista. Esto sin duda aumentaría la probabilidad de incorporación de nuevos profesionales en futuras ofertas públicas de empleo, al mismo tiempo que aumentaría la visibilidad de nuestro colectivo profesional en el conjunto de grupos profesionales de la Administración autonómica y por extensión, en el resto de entes relacionados, como organismos autónomos y empresas públicas, administración local, etc.

$\mathrm{f}$. Incorporemos en nuestros análisis, investigaciones, estudios, artículos, evidencia empírica, así como las últimas innovaciones teóricas y metodológicas. En esta materia el CAPS podría llevar a cabo actuaciones de formación interna en forma de seminarios o charlas impartidos por profesionales especializados. También podría inscribirse a fondos documentales y revistas especializadas en investigación social con los que trasladar al conjunto de miembros aquellos artículos y referencias relevantes en los diferentes ámbitos de actuación.

g. Exijámonos unos estándares de calidad mínimos en nuestras producciones en cuanto a referencias teóricas, metodológicas y de evidencia científica. En relación a esta estrategia, el CAPS podría contar con un grupo de voluntarios/lectores/ 
correctores que, a petición directa del profesional interesado, revisaran aquellos trabajos que se les presentaran.

h. Revaloricemos la sociología aplicada y descriptiva usada en la toma de decisiones basada en datos empíricos, tanto en el ámbito de la administración como de las empresas (propuesta e). En este sentido, proponemos la convocatoria de unos Premios Anuales (o Bienales) de Investigación Sociológica Aplicada y Comercial para galardonar y destacar aquella investigación de sociología descriptiva llevada a cabo en nuestra Comunidad Autónoma, independientemente de si han sido realizada por profesionales radicados en Asturias 0 no, que el CAPS considerara modélica en base a diferentes criterios (por establecer), entre los que cabría incluir la participación de al menos un sociólogo en el equipo de investigación.

i. Reivindiquemos la perspectiva sociológica especializada; a modo de colofón o resumen, como colectivo profesional debemos destacar la capacidad que otorga el conocimiento social experto para interpretar la construcción social de la realidad desde una perspectiva histórica y biográfica. Afirmar que los hechos sociales/colectivos no admiten interpretaciones individuales y que buena parte de los problemas individuales son construidos socialmente no es ni un descubrimiento ni una reivindicación corporativista. Sin menospreciar ni excluir a nadie, los sociólogos hemos culminado con éxito un largo proceso formativo que, por otra parte no acaba nunca, y que nos otorga en palabras de Mills (1969) la «mirada especializada global e interrelacional de los hechos sociales", complementaria a otras, no excluyente, y necesaria para un conocimiento de la realidad social con base empírica, más allá de la opinión y de la ideología.

\section{AGRADECIMIENTOS}

Queremos expresar nuestra gratitud a todas las personas que revisaron las versiones previas de este artículo, en particular a Manuel Fernández Esquinas, José Antonio Gómez-Yáñez y Arturo Lahera Sánchez, por su atenta lectura y sus sugerencias.

\section{REFERENCIAS BIBLIOGRÁFICAS}

Alvira, F. (2001). Presente y futuro de la sociología en España. RES. Revista Española de Sociología, 1, 49-59.

Bauman, Z. (1999). Modernidad líquida. Buenos Aires. Fondo de Cultura Económica.

Beck, U. (2001). Presente y futuro del Estado de Bienestar: el debate europeo. Buenos Aires: Miño y Dávila Editores.

Beck, U. (2002). La sociedad del riesgo global. Madrid: Ed. Siglo XXI.

Béteille, A. (2013). La vocación de la sociología. Una perspectiva pragmática. Diálogo Global, vol. 3 (2): 4-5. http://isa-global-dialogue.net/ wp-content/uploads/2013/07/v3i2-spanish. pdf, acceso 23 de diciembre de 2013.

Burawoy, M. (2005). Por una sociología pública. Política y Sociedad, 42 (1), 197-225.

Cipriani, R. (2012). La sociología en la Europa del Sur. RES. Revista Española de Sociología, 18: 97-103.

Cock, J. (2013). La vocación de la sociología. Desenmascarando la violencia lenta. Diálogo Global, vol. 3 (2): 6-7. http://isa-global-dialogue.net/ wp-content/uploads/2013/07/v3i2-spanish. pdf, acceso 23 de diciembre de 2013.

Colegio Oficial de Doctores y Licenciados en Ciencias Políticas y Sociología de Navarra (2008a). Perfil profesional de la sociología. Conociendo los perfiles profesionales de la sociología, nuevas oportunidades de empleo (en línea). http://www.cdn. sociologiac.net/2009/06/informeperfilesprofesionales.pdf, acceso15 de septiembre de 2016.

Colegio Oficial de Doctores y Licenciados en Ciencias Políticas y Sociología de Navarra (2008b). Entidades contratantes de profesionales de la Sociología. Conociendo los perfiles profesionales de la sociología, nuevas oportunidades de empleo (en línea). http://www.colsocpona.org/empresas09/empresas.html, acceso 15 de septiembre de 2016.

Davies, P. (2004). Is Evidence-Based Government Possible? Comunicación presentada en el 4th Annual Campbell Collaboration Colloquium, Washington D.C., 19 de febrero de 2004.

Del Campo, S. (dir.) (2001). Historia de la sociología española. Barcelona: Ariel. 
Diez-Nicolás, J. (2007). En el veinticinco aniversario de la Federación Española de Sociología. Revista Española de Sociología, 7, 89-97.

Federación Española de Sociología (2013). Acta de la Conferencia de Responsables Académicos del Área de Sociología de las Universidades Españolas. Presentada en la Facultat de Ciències Socials, Universitat de València. 21 y 22 de noviembre de 2013 (en línea). http://www.fes-sociologia. com/uploads/public/conferencia-responsablesacademicos/acta-reunion-noviembre-2013.pdf, acceso 15 de septiembre de 2016.

Fernández-Alcalde, J. B. (2010). La Sociología No Existe: Manifiesto o egiptización profesional. Intersticios. Revista Sociológica de Pensamiento Crítico, 4 (2), 5-27 (en línea). http://www. intersticios.es/article/view/6240/4344, acceso 23 de diciembre de 2013.

Gómez-Yáñez, J. A. (coord) (2010). Actas del Grupo de Trabajo N. ${ }^{\circ} 36$, La Sociología Como Profesión. Comunicaciones presentadas en el $X$ Congreso Nacional de Sociología de la FES, Pamplona.

Gómez-Yáñez, J. A. (2012). La Sociología Como Profesión. Revista Española de Sociología, 18: 125-130.

Grundiza, S. y López-Vilaplana, C. (2013). Intergenerational transmission of disadvantage statistics. EUROSTAT: Statistics in focus 27/2013 (en línea). http://epp.eurostat.ec.europa.eu/ statistics_explained/index.php/Intergenerational_transmission_of_disadvantage_statistics, acceso 15 de septiembre de 2016.

Guilló, C. (2007). De la Sociología precaria y clandestina a la Sociología crítica y transformadora. RES. Revista Española de Sociología, 7: 77-87.

Lichtenstein, B. (2013). ¿Está en crisis la sociología norteamericana? Diálogo Global, vol. 3 (2): 31-32.

Machado, P. (2012). Retos de la profesionalización de la sociología en contexto de crisis. RES. Revista Española de Sociología: 18, 107-120.

Mills, Ch. W. (1969). La imaginación sociológica. México: Fondo de Cultura Económica.

Ortí, A. (2007). Veinticinco años después: el oficio de sociólogo en la España plural. Revista Española de Sociología, 7: 27-75.
Pérez-Yruela, M. (2007a). Los primeros pasos de la Federación Española de Sociología. Revista Española de Sociología, 7: 113-117.

Pérez-Yruela, M. (2007b). El retorno de la sociología. Revista Española de Sociología, 7: 13-26.

Pérez-Yruela, M. (2011). La sociología hace treinta años, la sociología dentro de treinta años: Clausura del X Congreso de Sociología. Revista Española de Sociología, 15: 135-146.

Robertson, R. (1992). Globalization. Social Theory and Global Culture. London: Sage Publications. Rodríguez-lbáñez, J. E. (2011). La sociología española, 1959-2009. Un análisis generacional. Documentos de Trabajo: Sociología en Lengua Castellana (DTSC), 1: 1-4.

Subirats, M. (2007). Sociología y Política, una relación siempre problemática. RES. Revista Española de Sociología, 7: 99-110.

Tezanos, J. F. (2001). Reflexiones sobre el presente y el futuro de la sociología. RES. Revista Española de Sociología, 1: 33-48.

Valdera, J. M. (2011). Las partes formales de la Sociología desde la Teoría del Cierre Categorial: una aproximación analítica. El Catoblepas. Revista crítica del presente, 114, 9.

Woolcock, M. (2001). La importancia del capital social para comprender los resultados económicos y sociales. Conferencia impartida en la Facultad de Ciencias Sociales Universidad de La República - Uruguay.

\section{NOTA BIOGRÁFICA}

Ángel Alonso Domínguez, doctor por la Universidad de Oviedo, experto universitario en Dirección de RRHH y licenciado en Sociología por Ia UNED. Ha desarrollado su labor profesional en la empresa privada durante más de veinte años, en diversos ámbitos como el marketing, el sector naval, las organizaciones sindicales o la consultoría. Actualmente es, además, profesor asociado del Departamento de Sociología de la Universidad de Oviedo y profesor contratado en la Facultad Padre Ossó de 0viedo. Ha realizado investigaciones y publicaciones sobre transiciones laborales, innovación y consumo. Ha sido miembro de la Red 
de Excelencia Reconciling Work and Welfare in Europe (RECWOWE) y forma parte del grupo de investigación Promoviendo el Empleo y el Bienestar en Europa (PROMEBI: http:// www.unioviedo.es/ promebi/). Es presidente de la Asociación Asturiana de Sociología (2012-Actualidad) y vocal del Colegio Oficial de Ciencias Políticas y Sociología del Principado de Asturias.

Nerea Eguren Adrián, licenciada en Sociología por la U.C.M. especialista universitario en Investigación Social y de Mercados (U.C.M) y experto universitario en Evaluación de Programas Sociales (U.0). Ha desarrollado su labor profesional en el ámbito de políticas de bienestar social. Dentro del Gobierno del Principado de Asturias ha sido asesora técnica del consejero de Asuntos Sociales y responsable del Área de Planificación y Sistema de Información de la Consejería de Bienestar Social. En la actualidad es directora general de Planificación, Ordenación e Innovación Social de la Consejería de Servicios y Derechos Sociales. También ha sido profesora asociada de la Universidad de Oviedo y docente en diversos Máster y títulos propios de la misma. Fue presidenta de la Asociación Asturiana de Sociología (2007-2012) y vicedecana del Colegio Asturiano de Ciencias Políticas y Sociología (2015).

Antón González Fernández, licenciado en Sociología por la Universidad Complutense de Madrid (1983-1988), realizó cursos de doctorado en la Universidad de Oviedo (1989-1991) y formación de postgrado en medioambiente (curso de Contaminación e Ingeniería Ambiental de la U. de Oviedo 1990-1991 y Máster en Gestión Medioambiental del Instituto de Investigaciones Ecológicas y The Open International University 1994-1995). Entre 1996 y 2006 trabajó como agente de Desarrollo Local y desde ese último año en la administración del Principado de Asturias donde en la actualidad es el responsable del Observatorio sobre Drogas de Asturias. Ha realizado y/o participado en estudios y publicaciones sobre desarrollo local, juventud y salud pública e impartido formación para diversas empresas e instituciones en temas de desarrollo local, medio ambiente, empleo y con- sumo de drogas. Es secretario del Colegio Oficial de Ciencias Políticas y Sociología del Principado de Asturias.

\section{ANEXO 1. METODOLOGÍA DE PARTICIPACIÓN Y ELABORACIÓN DEL TRABAJO}

\section{Fase 1. Constitución del grupo de trabajo y elaboración del primer borrador}

\subsection{Constitución del Grupo de Trabajo. Junio de 2013}

La relación de las personas interesadas en colaborar inicialmente con el grupo de trabajo son:

Ángel Alonso Domínguez

Arsenio Valbuena

Cecilia Díaz-Méndez

Margarita Eguiagaray

María González Ramos

Damián Herrera Cuesta

Sandra Losada Menéndez

Carmen Suárez Lombraña

Irene Tejedor Rodríguez

Actúan como coordinadores Antón González Fernández y Nerea Eguren Adrián

1.2. Lecturas y elaboración de propuestas. Del 1 de julio al 15 de septiembre de 2013

En esta fase se proponen una serie de lecturas mínimas sobre la sociología como profesión, con el objeto de que todo el grupo de trabajo compartiera un marco teórico-conceptual común. Estas lecturas no supusieron ningún límite, de manera que quedó abierta la posibilidad de enriquecer con otras aportaciones esta base documental. El equipo coordinador se encargó de recabar la documentación y enviarla al resto de miembros del grupo, que también realizó aportaciones documentales.

Tras este periodo, se propuso que cada una de las personas integrantes del grupo de trabajo elaborase un texto de ideas que incluyera los conceptos que consideraran centrales en su planteamiento, con un breve desarrollo de los mismos. Este 
resumen debía ser lo más claro y operativo posible (máximo 300 palabras) y sería enviado a los coordinadores del Grupo quienes lo distribuirían entre el resto de miembros.

Los miembros del grupo de trabajo que enviaron un documento escrito con sus reflexiones son:

\section{Ángel Alonso Domínguez \\ Nerea Eguren Adrián \\ Antón González Fernández \\ María González Ramos \\ Damián Herrera Cuesta \\ Sandra Losada Menéndez}

Otro miembro del mismo, Margarita Eguiagaray, envió documentación relacionada.

\subsection{Elaboración del primer borrador. Del 15 al 30} de septiembre 2013

Durante este período los coordinadores realizaron un texto resumen de las propuestas principales que, posteriormente, se remitió a los miembros del grupo.

\section{Fase 2. Debate secuencial de los diferentes borradores}

2.1. Reunión del grupo de trabajo sobre el primer borrador. 15 octubre de 2013

Con fecha 15 de octubre se produce el primer encuentro presencial de los coordinadores y miembros del Grupo del Trabajo con el objetivo de debatir el documento que recoge las propuestas elaboradas hasta el momento y acordar las bases de un primer borrador del documento "La sociología como Profesión».
2.2. Aportaciones del resto asociados/as. Del 20 de octubre al 11 de noviembre de 2013

A través de la lista de distribución de la AAS se ofrece al conjunto de personas asociadas la posibilidad de conocer el texto elaborado hasta el momento para que realicen aportaciones al mismo.

2.3. Elaboración del borrador definitivo. Del 12 al 26 de noviembre de 2013

Una vez recogidas todas las sugerencias recibidas de la fase anterior, los coordinadores elaboran un texto que será el marco previo que definirá el documento sobre "La Sociología como Profesión».

2.4. Presentación del borrador dentro del foro de encuentro y debate "Coloquio-cena" que organiza la AAS. 28 de noviembre de 2013

Los coordinadores presentaron el documento en el 10. ${ }^{\circ}$ coloquio de este ciclo, con el objetivo de recibir aportaciones al mismo y elaborar el documento final.

\section{Fase 3. Presentación del documento final. Diciembre 2013 - agosto 2014}

Con las aportaciones recibidas en el acto anterior, los coordinadores junto con Ángel Alonso, elaboran el documento final sobre la Sociología como Profesión. A través de la lista de distribución de la AAS se ofrece al conjunto de persona asociadas la posibilidad de conocer el texto en esta última fase y realizar aportaciones al mismo. Por último, se envía el manuscrito a reconocidos expertos en el ámbito de la Sociología, fuera del ámbito territorial asturiano, de los cuales se obtienen, también, las últimas sugerencias al documento. 


\section{ANEXO 2. IDEAS PRINCIPALES DEL DEBATE}

Tabla 1. Análisis DAFO

\begin{tabular}{l} 
Debilidades \\
\hline 1. Alejamiento de los problemas que preocupan \\
a los ciudadanos. Supremacía de la sociología \\
"académica".
\end{tabular}

2. Debilidad práctica. Baja estima de la sociología aplicada 0 descriptiva.

3. Carencia de técnicas, metodologías y "paradigmas teóricos" innovadores.

4. Pequeño tamaño del mercado de trabajo de Asturias.

5. Escasa conciencia como colectivo profesional y escasa corporativización "positiva».

6. Atomización, dispersión, soledad de los profesionales.

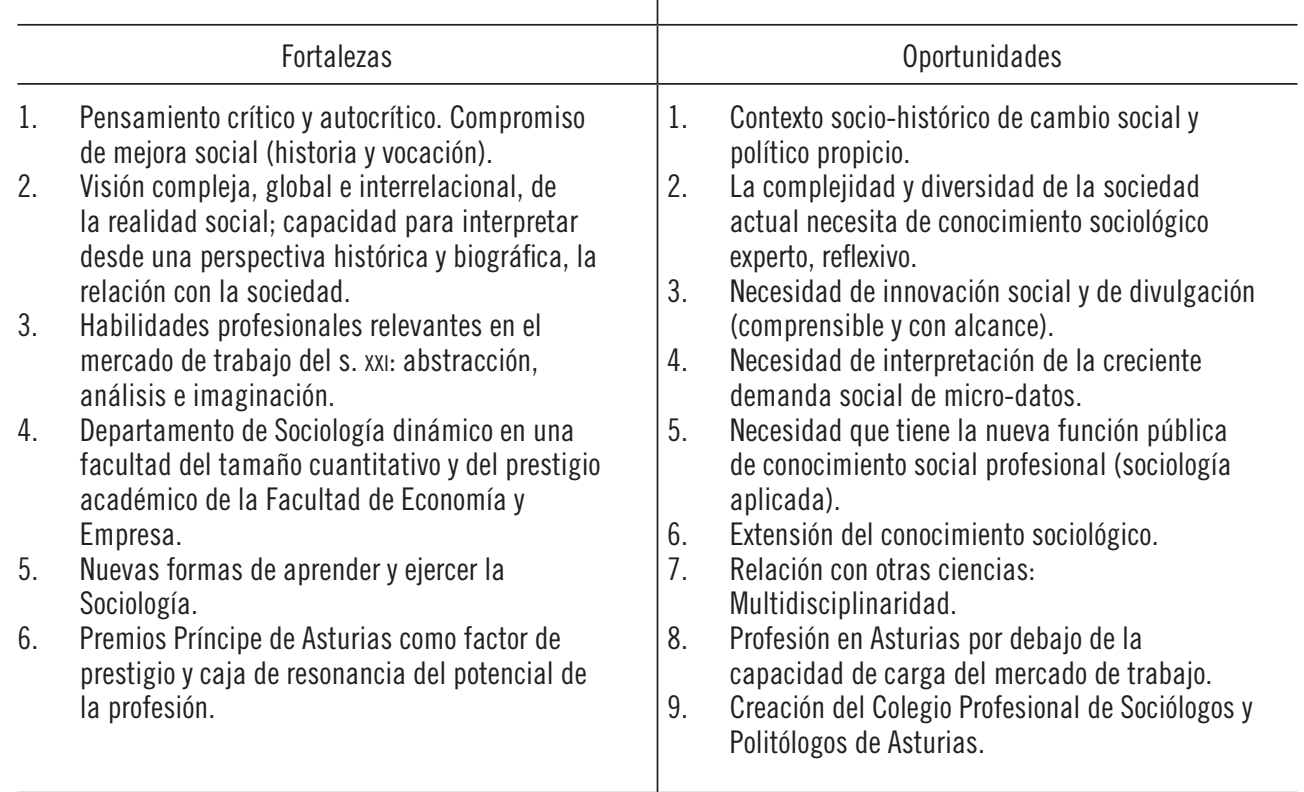

1. Corriente ideológica liberal (primacía de las explicaciones individuales, aisladas, frente a las colectivas) que relega la sociología como disciplina científica.

2. Irrupción de nuevos ámbitos profesionales (marketing, consumo, intervención social) que colonizan el terreno académico y profesional.

3. Popularización de la perspectiva sociológica; pérdida de identidad profesional, prestigio y rigor científico.

4. Escasa presencia en la administración pública asturiana y con indefinición de funciones. 